\title{
Status of MuSIC facility
}

\author{
Yuki Matsumoto*i \\ Osaka University \\ E-mail: $\mathrm{y}$-mat sumotodkuno-g.phys.sci.osaka-u.ac.ip
}

The MuSIC is a DC muon beam facility under the Research Center of Nuclear Physics (RCNP), Osaka University. The MuSIC has a 3.5 Tesla Pion capture solenoid and a $36^{\circ}$ curved 2 Tesla transport solenoid. These solenoids enable the MuSIC to get high muon production efficiency per proton, expected about $2 \times 10^{8}\left[\mu^{+} / \mathrm{s} / \mu \mathrm{A}\right]$. This efficiency is 1000 times higher than that of conventional muon beam facilities.

In 2014 the new muon beam line was constructed. This beam line is enable to focus beam and select momentum and particle by some magnets. This beam line was designed for measurement of $\mu \mathrm{SR}$ or muonic X-ray. These measurement request $10^{5}\left[\mu^{+} / \mathrm{s} / \mu \mathrm{A}\right]$ for positive muon and $10^{4}\left[\mu^{+} / \mathrm{s} / \mu \mathrm{A}\right]$ for negative muon. The beam line aims at these muon yield.

In this paper the author describes detail design and prospects of the MuSIC including the new beam line.

16th International Workshop on Neutrino Factories and Future Neutrino Beam Facilities 25 -30 August, 2014

University of Glasgow, United Kingdom

* Speaker.

${ }^{\dagger}$ A footnote may follow. 


\section{Introduction}

The MuSIC is a high intensity muon beam facility under RCNP, Osaka University (Figure $\mathbb{D}$ ). The proton beam is provided by the RCNP' $\mathrm{s}$ ring cyclotron. The proton beam power is $400 \mathrm{~W}$. The MuSIC achieves a muon intensity of $10^{8}\left[\mu^{+} / \mathrm{s}\right]$. Compared with that, the the Paul Sherrer Institute (PSI) achieve a same muon intensity with $1.3 \mathrm{MW}$ proton beam. This shows that the muon production efficiency of the MuSIC is over 1000 times higher than that of PSI. The MuSIC has two features.

- Pion capture system : The pion capture system is a system involves a pion capture solenoid and a $36^{\circ}$ curved transport solenoid. Protons enter the pion capture solenoid and hit a graphite target. Pions produced from protons are captured by a magnetic field of the pion capture solenoid and the $36^{\circ}$ curved transport solenoid transport these pions. Pions decay to muons while transported.

- MuSIC M1 Beam Line : MuSIC M1 Beam Line has SLITs(SL), Staring Magnets(STH), Quadrupole Magnets(QM), Bending Magnets(BM) and a Spin Rotator(SR). Particles transported by the $36^{\circ}$ curved transport solenoid enter the MuSIC M1 Beam Line. While passing through the beam line, focused muons with uniform momentum are taken out.

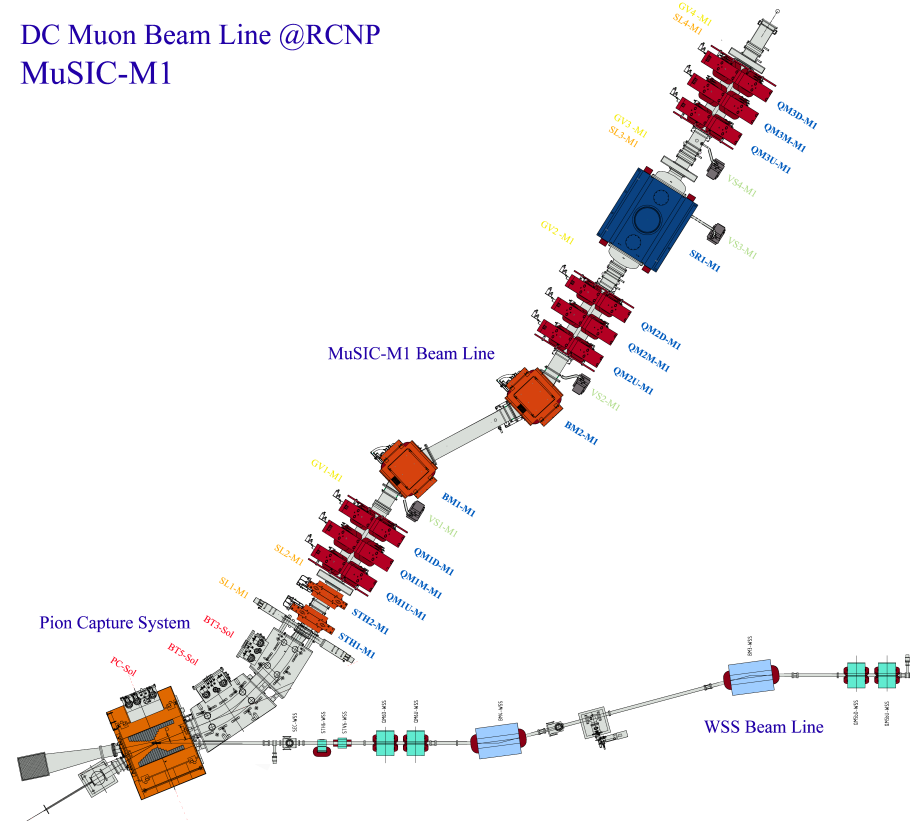

Figure 1: Layout of MuSIC. Protons emitted from the WSS Beam Line enter the Pion Capture System and hit graphite target. Pions produced from protons are captured by the pion capture solenoid and transported by the $36^{\circ}$ curved transport solenoid. The pions decay to muons in the transport magnet and muons are corrected by the MuSIC M1 beam Line.

\section{Pion capture system}

The construction of the pion capture system was completed in JFY2009. In 2010 measurement 
proved that the MuSIC achieves about $10^{8}[\mu / \mathrm{s} / \mu \mathrm{A}]$ muon production efficiency. This is 1000 times higher than that of PSI and TRIUMF. The details are described in this section.

\subsection{Features}

A special structure of the pion capture system is enable the MuSIC to achieve a high muon production efficiency. In the case of the conventional muon beam facility, the neutron facility uses same proton beam line. So the structure of muon beam facility should be like a Figure $\square$. The target is thin and the pion capture solenoid is set beside the target. In contrast, the MuSIC can use full proton beam. Figure [] shows the structure of the MuSIC pion capture system. A $200 \mathrm{~mm}$ thick graphite target stops the protons, so a large mount of pions are produced. Pions are captured by the pion capture solenoid surrounding the graphite target over a large solid angle. It makes pion production and capture efficiency higher. Pions captured by the pion capture solenoid are transported by the $36^{\circ}$ curved transport solenoid and decay to muons. A $2 \mathrm{~T}$ solenoid magnet and a $0.04 \mathrm{~T}$ dipole magnet select muon charge and momentum 30-50 MeV/c. Thus the MuSIC obtains a high muon production efficiency.
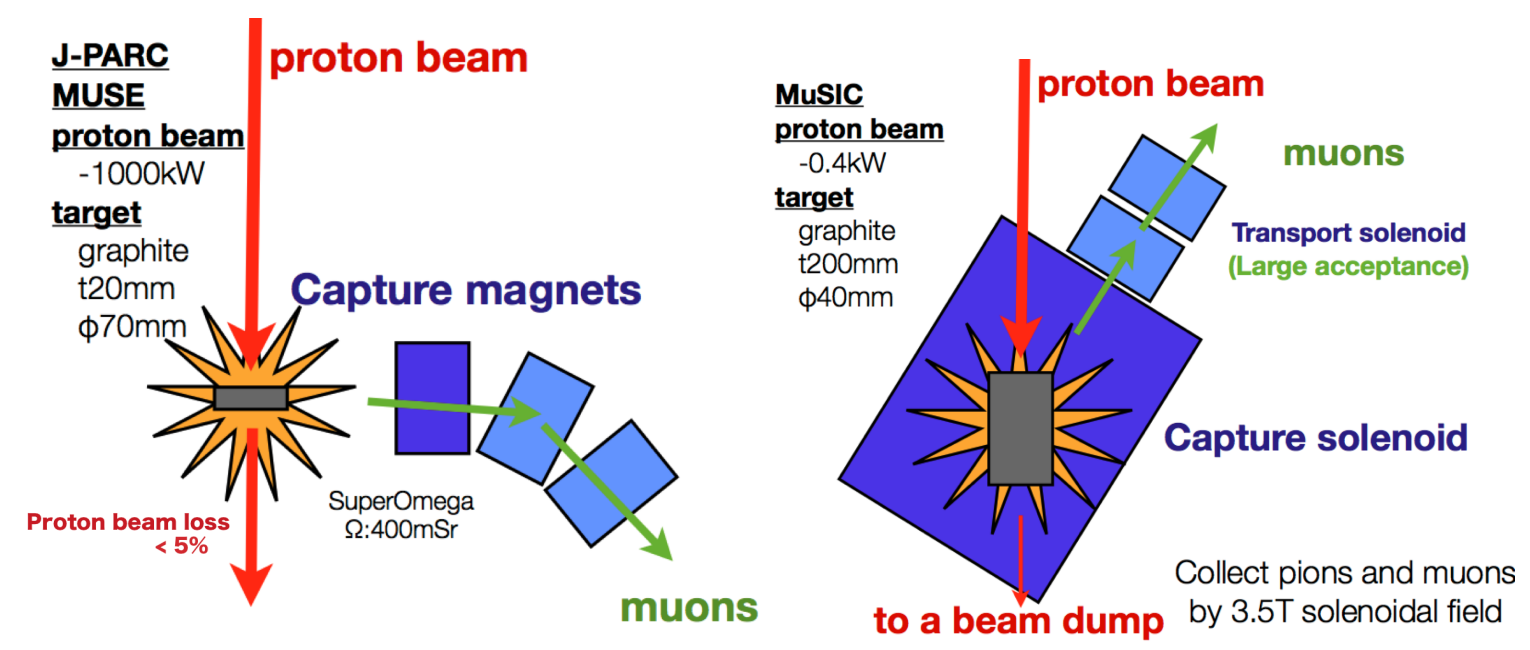

\section{to the neutron facility}

Figure 3: The pion capture system of MuSIC. The thick target stops a large amount of protons and protons produce

Figure 2: A conventional muon beam facility. The neutron pions. Pions are captured by the pion capture solenoid surfacility uses same proton beam, so the target is thin and the rounding the graphite target over a large solid angle. pion capture solenoid is set beside the target.

\subsection{G4beamline}

G4beamline is a simulator based on Geant 4 [四]. The MuSIC structure from the graphite target to the $36^{\circ}$ curved transport solenoid were described by G4beamline. $392 \mathrm{MeV}$ initial protons were used in G4beamline simulation. The result shows features of muons at the exit of the $36^{\circ}$ curved transport solenoid. Figure $⿴ 囗 十$, show the result of a positive muon flux and momentum distribution when the $36^{\circ}$ curved transport solenoid select positive muons. Figure 1 shows the beam size will be about $300 \mathrm{~mm}$. Figure 5 shows a peak at $60 \mathrm{MeV} / \mathrm{c}$ and the existence of surface muons. Figure 6 , $\square$ show the result of a negative muon flux and momentum distribution when the $36^{\circ}$ curved transport 
solenoid select negative muons. Figure $\mathbf{6}$ shows the beam size will be about $300 \mathrm{~mm}$. Figure $\square$ shows a peak at $60 \mathrm{MeV} / c$. The new muon beam line was designed based on these results.

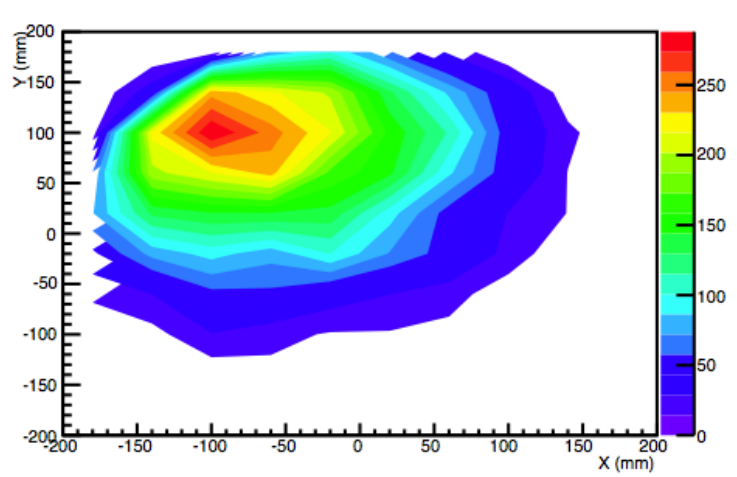

Figure 4: The positive muon flux at the $36^{\circ}$ curved transport solenoid

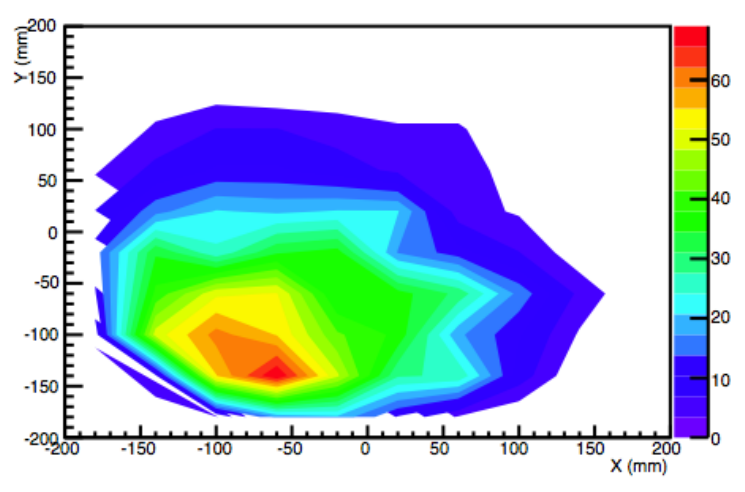

Figure 6: The negative muon flux at the $36^{\circ}$ curved transport solenoid

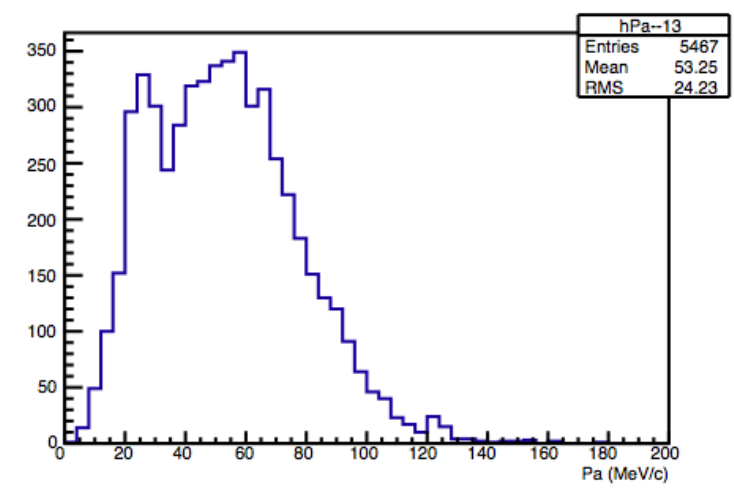

Figure 5: The positive muon momentum distribution at the $36^{\circ}$ curved transport solenoid

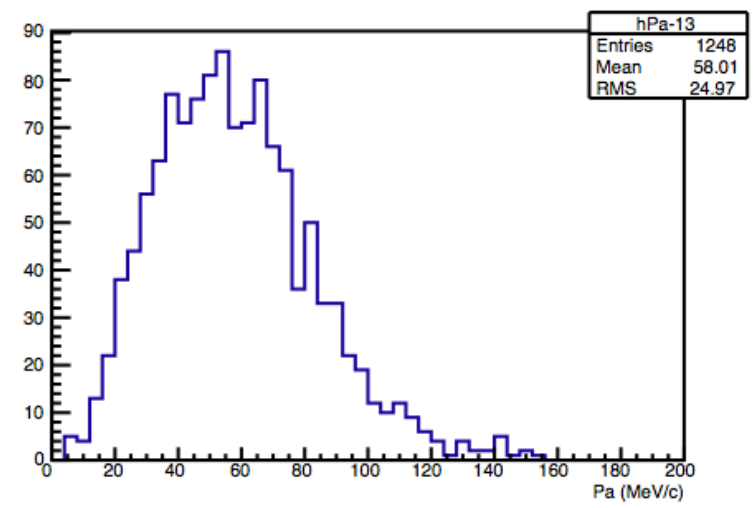

Figure 7: The negative muon momentum distribution at the $36^{\circ}$ curved transport solenoid

\subsection{Measurement of muon yield}

In 2011 two measurement of muon yield was done to confirm the performance of the pion capture system. One was measurement of muon lifetime to measure a positive muon yield, second was measurement of muonic X-ray to measure a negative muon yield. A target to stop muons was placed at the exit of the $36^{\circ}$ curved transport solenoid and two plastic scintillators $\left(380 \times 50 \times 3.5 \mathrm{~mm}^{3}\right)$ were placed on either side of the target. Plastic scintillators made triggers and detect electrons decayed from stopping muons for measurement of muon lifetime. And Ge detector was placed to measure muonic X-rays. Figure $\mathbb{Q}$ shows the result of the measurement of muon lifetime and Figure $\mathbb{Q}$ shows the result of the measurement of muonic X-ray. Table $\mathbb{W}$ shows comparisons of results of G4beamline and measurement. It indicates that the results of measurement correspond to that of G4beamline within order and the pion capture system has same performance as designed. 

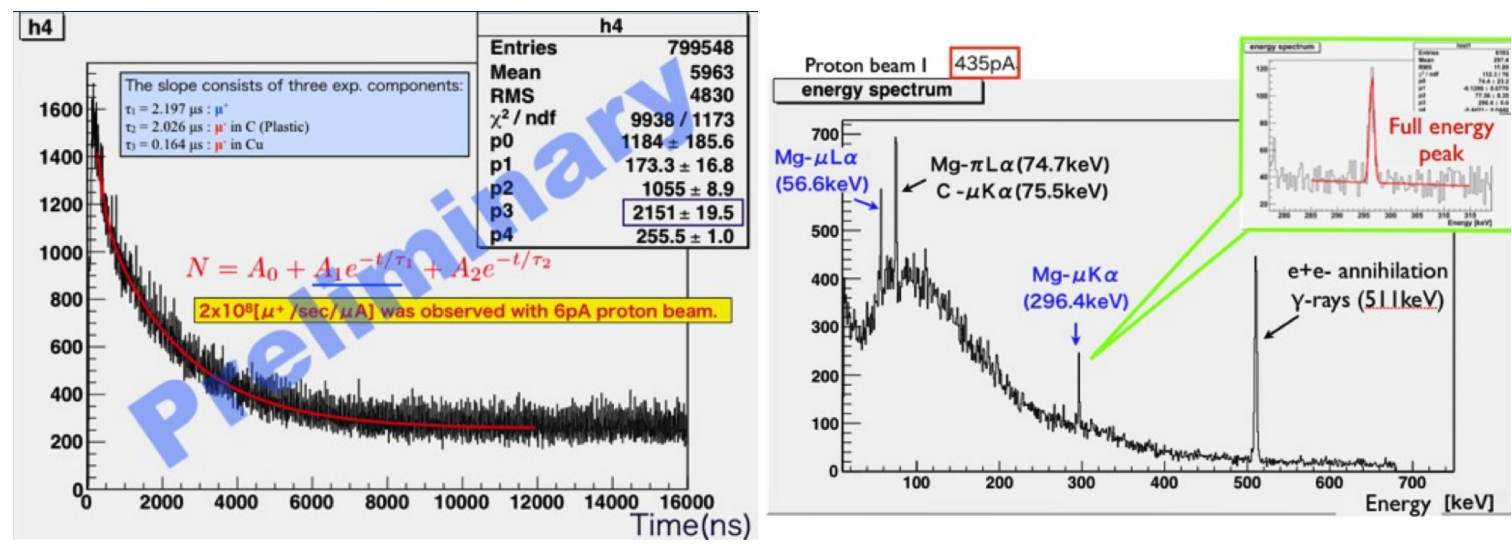

Figure 8: The result of measurement of muon lifetime. An analysis showed that positive muon yield was $3 \times 10^{8}$ $\left[\mu^{+} / \mathrm{s} / \mu \mathrm{A}\right]$.

Figure 9: The result of measurement of muonic X-ray. An analysis showed that positive muon yield was $(1.7 \pm 0.3) \times$ $10^{8}\left[\mu^{+} / \mathrm{s} / \mu \mathrm{A}\right]$.

Table 1: The comparison of G4beamline and measurement.

\begin{tabular}{|c|c|c|}
\hline & Simulation & measurement \\
\hline$\mu^{+}$ & $2 \times 10^{8}\left[\mu^{+} / \mathrm{s} / \mu \mathrm{A}\right]$ & $3 \times 10^{8}\left[\mu^{+} / \mathrm{s} / \mu \mathrm{A}\right]$ \\
\hline$\mu^{-}$ & $1.4 \times 10^{8}\left[\mu^{+} / \mathrm{s} / \mu \mathrm{A}\right]$ & $(1.7 \pm 0.3) \times 10^{8}\left[\mu^{+} / \mathrm{s} / \mu \mathrm{A}\right]$ \\
\hline
\end{tabular}

\section{MuSIC M1 Beam Line}

In 2014 the new muon beam line (MuSIC M1 Beam Line) was constructed. In the end of 2014 a commissioning will be done. This beam line was designed for measurement of $\mu$ SR or muonic $\mathrm{X}$-ray. Details of the new beam line is described below using symbols in Figure $\mathrm{W}$. Particles emitted from the exit of the $36^{\circ}$ curved transport solenoid are guided to the beam line by Staring Magnets (STH1, STH2). At that time, emittance of beam is determined by SLITs(SL1, SL2). Each SLITs move $\mathrm{x}$ and $\mathrm{y}$ axis and cut beam two-dimensionally. Particles passed through SL2 are focused by a Triplet Quadrupole Magnets (QM1). Bending Magnets (BM1, BM2) select momentum of particles passed through QM1. Then particles are focused by a Triplet Quadrupole Magnets (QM2). A spin Rotator (SR) rotates spin of particles and select muons. A SLIT(SL3) determines the purity of muon and the spread of momentum. At last a Triplet Quadrupole Magnets (QM3) and a SLIT(SL4) determine the beam size.

\subsection{Spin Rotator}

A Spin Rotator is an apparatus also called DC separator or wine filter. It rotates a spin of particle and select particle by magnetic field and electric field. Figure $\mathbb{}$ l shows the principle. Momentum of particles enter the Spin rotator is same because they passed through Bending Magnet. Magnetic field bend a trajectory of charged particle which have same momentum with a certain curvature. In contrast, electric field bend a trajectory of charged particle with a curvature dependent on a mass of particle. By using this effect, particle is selected. Spin of particle is rotated by 
magnetic field. The Spin Rotator set on MuSIC M1 Beam Line has a $1.8 \mathrm{~m}$ long electrode and a space of electrodes is $0.15 \mathrm{~m}$. Maximum supply Voltage is $\pm 400 \mathrm{kV}$ on design. This power rotate spin of muon $(\mathrm{p}=27 \mathrm{MeV} / \mathrm{c}) 80^{\circ}$. Now maximum supply Voltage achieves at $\pm 380 \mathrm{kV}$ with $\mathrm{Ar}$ gas selling.

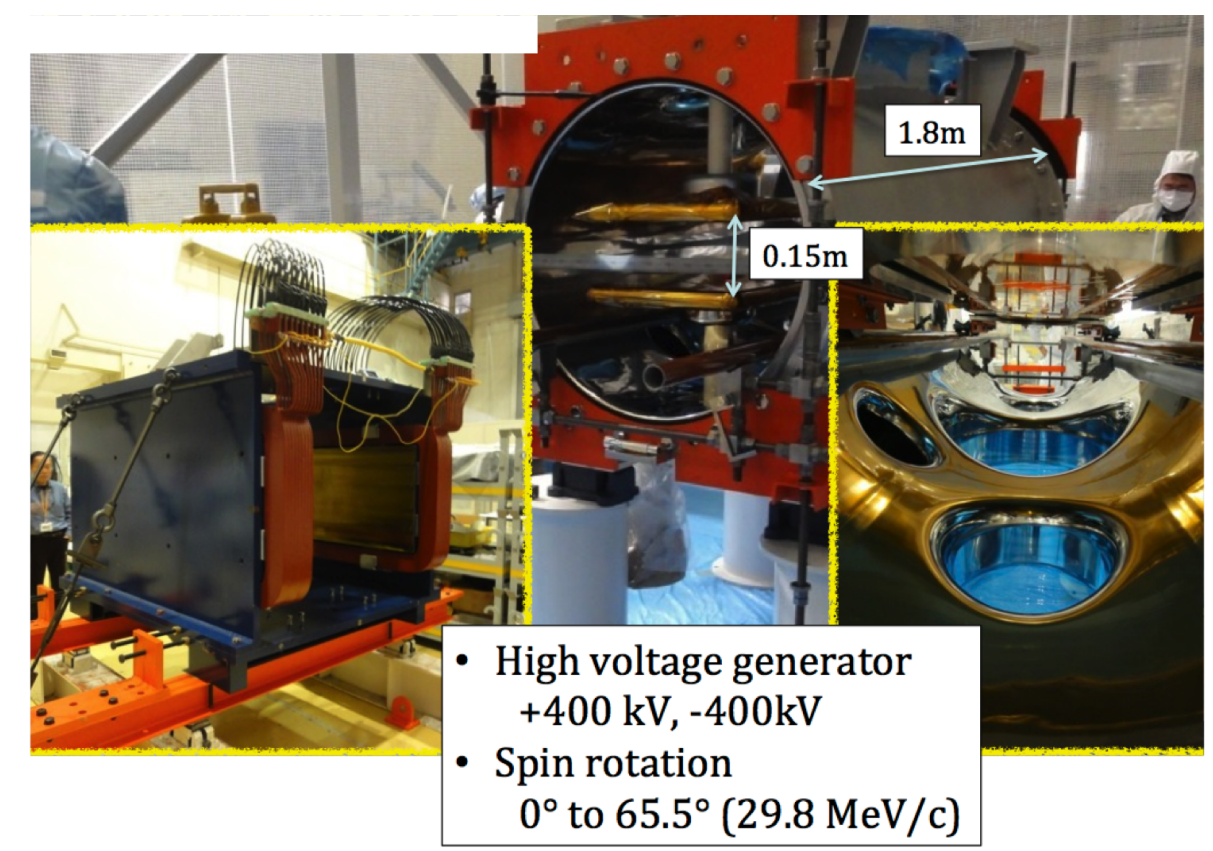

Figure 10: Spin Rotator. An electrode is $1.8 \mathrm{~m}$ long and a space of electrodes is $0.15 \mathrm{~m}$.

\subsection{Beam line optics}

Transport and TURTLE [ [ $]$ ] was used to design the beam optics. The beam profile of the MuSIC M1 Beam Line was calculated by Transport and TURTLE. Figure $\mathbb{\square}, \mathbb{Z}$ shows the result of beam profiles in an $\mathrm{X}$-axis direction and in an Y-axis direction at the exit of the beam line. Table $\square$ shows the initial state of the calculation at the exit of the $36^{\circ}$ curved transport solenoid. The result indicate that the beam size will be smaller than $3 \mathrm{~mm}$ (RMS) and about $78 \%$ muons will pass through the beam line.

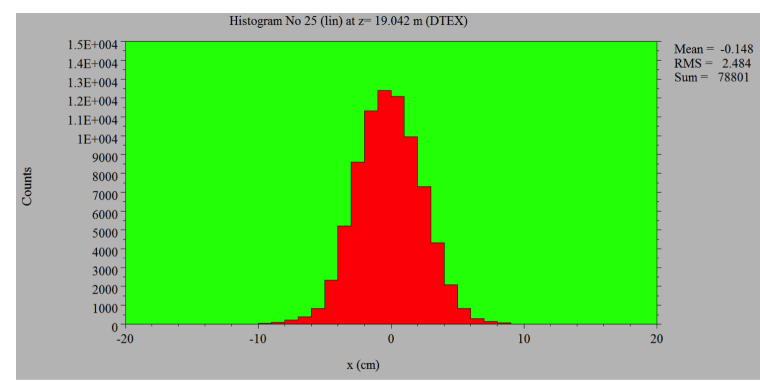

Figure 11: The beam profiles in an $X$-axis direction calcu- Figure 12: The beam profiles in an $X$-axis direction calculated by Transport and TURTLE.

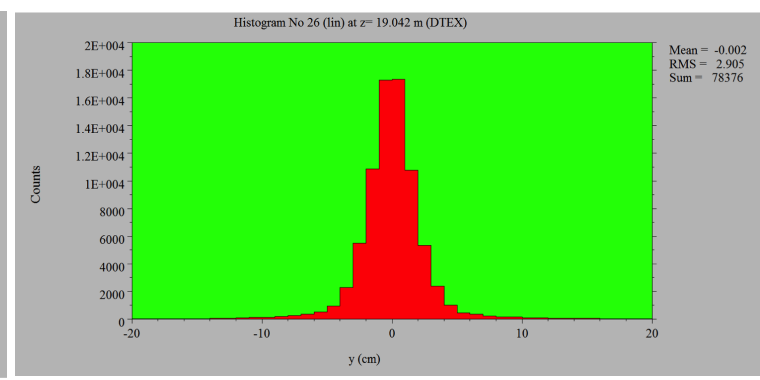

lated by Transport and TURTLE. 
Table 2: The initial state of the calculation at the exit of the $36^{\circ}$ curved transport solenoid. $\delta$ depends on gaussian.

\begin{tabular}{|c|c|c|c|c|c|c|}
\hline$\mu$ runs & $\delta \mathrm{x}$ & $\delta \theta_{x}$ & $\delta \mathrm{y}$ & $\delta \theta_{y}$ & $\mathrm{p}$ & $\Delta \mathrm{p} / \mathrm{p}$ \\
\hline 100000 & $2 \mathrm{~cm}$ & $50 \mathrm{mdad}$ & $2 \mathrm{~cm}$ & $50 \mathrm{mrad}$ & $29.8 \mathrm{MeV} / c$ & $1 \%$ \\
\hline
\end{tabular}

\section{Conclusion}

The MuSIC under the RCNP, Osaka-University introduces the pion capture system. In this system, a $200 \mathrm{~mm}$ thick graphite target is placed in a pion capture solenoid. This special structure enable the MuSIC to achieve high muon production efficiency. In 2011 measurement of muon yield indicated that the muon production efficiency of the MuSIC was over 1000 times higher than that of conventional muon beam facilities.

In 2014 the new muon beam line was constructed. This beam line was designed for measurement of $\mu \mathrm{SR}$ or muonic X-ray. This beam line is enable to make focused and pure muon beam with uniform momentum. Calculations of Transport and TURTLE indicated the beam size at the exit of the beam line will be smaller than $3 \mathrm{~mm}$ and $78 \%$ muons will pass through the beam line. The end of 2014, a commissioning of the beam line will be done.

\section{References}

[1] http://G4beamline.muonsinc.com

[2] U. Rohrer, PSI Graphic Transport/Turtle, based on CERN-SLAC-FNAL version by K. Brown et al. 